\title{
Versorgung von Migranten
}

\author{
Meryam Schouler-Ocak, Marion C. Aichberger
}

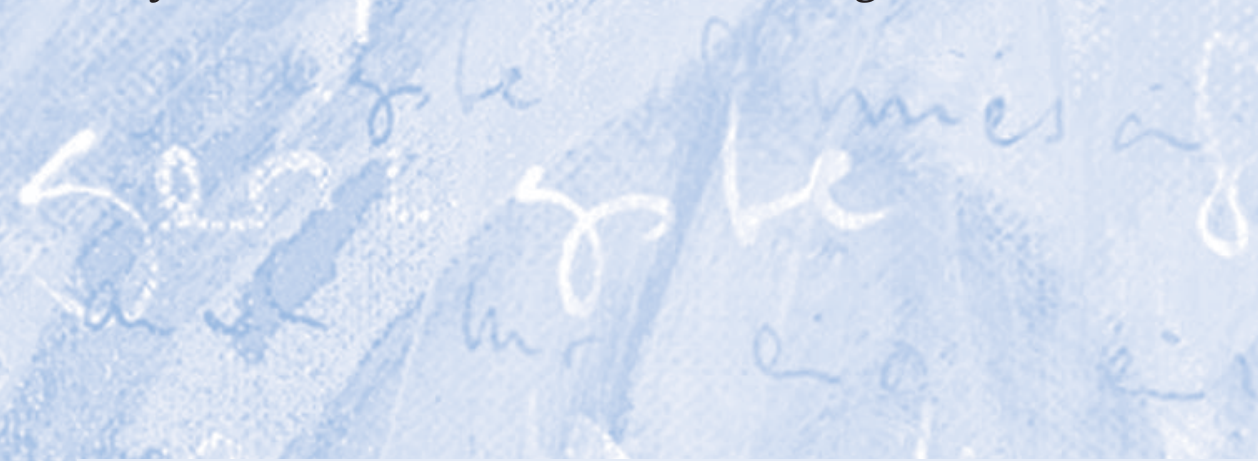

Migranten haben besondere gesundheitliche, psychosoziale und ökonomische Belastungen. Bei ihnen beeinflussen kulturelle, migrationsspezifische und soziale Faktoren die Prävalenz, Manifestation und den Verlauf psychischer Erkrankungen.

\section{Einleitung}

Migrationserfahrungen, Migrationshintergrund oder kulturelle Normen sind für die meisten Psychiater, Psychotherapeuten und Psychologen keine unbekannten Begriffe mehr. Zwar sind Verständnis und Akzeptanz gestiegen für die Bedeutung soziokultureller Einflussfaktoren auf die Krankheitsdarbietung, die Krankheitsverarbeitung, für die Diagnostik, die Therapie und die Prävention, doch in der Praxis scheitert es noch immer an der Umsetzung.

So ist es bislang im klinischen Alltag oft schwierig, die migrations- bzw. kulturspezifischen Aspekte und deren Stellenwert für die Genese und den Verlauf im Einzelfall adäquat einzuschätzen. Sprachbarrieren [31, 50], Unterschiede in der Präsentation von psychischen Beschwerden [43, 44], Differenzen in der Erwartung bez. Diagnostik und Therapie zwischen Patienten und Behandler können z.T. schwer überwindbare Hürden im Behandlungsalltag bilden [69]. Ein Abbau dieser Barrieren mit der Zeit erfordert auch die Aufnahme des Themas in Aus- und Weiterbildung. In diesem Beitrag werden die Begriffe Migranten bzw. kulturelle Minderheit verwendet, wenn Menschen mit Migrationshintergrund gemeint sind.

\section{Definition}

Mensch mit Migrationshintergrund bezeichnet alle Menschen, die „... nach 1949 auf das heutige Gebiet der Bundesrepublik Deutschland zuwanderten, sowie alle in Deutschland geborenen Ausländer und alle in Deutschland als Deutsche Geborenen mit zumindest einem zugewanderten oder als Ausländer in Deutschland geborenen Elternteil..." [94].

\section{Migrationsgeschichte}

Deutschland entwickelte sich erst seit Mitte der 1950er-Jahre zu einem der wichtigsten europäischen Einwanderungsländer [20]. Es wurden zunächst „Gastarbeiter“ aus Italien, später aus Spanien, Portugal, Griechenland, dem ehemaligen Jugoslawien und aus der Türkei angeworben. Nach 1973, dem Jahr des Anwerbestopps, veränderte sich der Zuzug von Ausländern. Es folgte der Zuzug von Familienangehörigen, und seit den 1980er-Jahren kamen zudem Bürgerkriegsflüchtlinge und Asylbewerber sowie deutschstämmige Bürger der Sowjetunion und später der GUSStaaten nach Deutschland. Auch die Globalisierung und die weltweit zunehmende Mobilisierung trugen dazu bei, dass Menschen ihren Lebensmittelpunkt nach Deutschland verlagerten. 2001 stellte die Regierungskommission zur Zuwanderung unter Leitung von Rita Süssmuth fest, dass Deutschland ein Einwanderungs- 
land geworden ist (Süddeutsche Zeitung vom 5.7.2001, S.8).

\section{Aktuelle statistische Daten}

Obwohl die Nachkommen der 1. Generation von Einwanderern größtenteils in Deutschland geboren sind und selber keine Migrationserfahrung haben, werden sie auch zu Personen mit Migrationshintergrund gerechnet. Bis 2005 wurde zwischen Deutschen und Ausländern unterschieden. Erst der Mikrozensus 2005 differenzierte zwischen Personen mit und ohne Migrationshintergrund (s.o. Definition) [93]. Demnach hatten 2005 18,6\% (15,3 Millionen) der Gesamtbevölkerung einen Migrationshintergrund, darunter waren 8,9\% (7,4 Millionen) Ausländer [93]. Der Mikrozensus 2013 zeigte, dass sich der Anteil an Personen mit Migrationshintergrund auf nun mehr als 20,5\% (16,5 Millionen) der Gesamtbevölkerung erhöht hat [94]. Personen mit Migrationshintergrund sind im Durchschnitt deutlich jünger als die ohne Migrationshintergrund (35,2 vs. 46,7 Jahre) [94]. Bei den unter 5-Jährigen haben demnach inzwischen 34,5\% (1172 000 Personen) einen Migrationshintergrund [94].

\section{Herkunft der Migranten in Deutschland}

- 36,6\% (ca. 5,4 Mio.) aus den 28 Mitgliedsländern der Europäischen Union

- 17,6\% (ca. 2,7 Mio.) aus der Türkei

- 9,6\% (ca. 1,5 Mio.) aus Polen

- 8,8\% (ca. 1,4 Mio.) (Spät-)Aussiedler aus den Nachfolgestaaten der ehemaligen Sowjetunion

- 7,5\% (ca. 1,1 Mio.) aus der Russischen Föderation

- 5,7\% (903000) aus Kasachstan

- 4,9\% (783 000) aus Italien [94]

\section{Erklärungsmodelle und Behandlungserwartung}

\section{Kultureller Kontext}

Bei den oben genannten Daten wird schnell deutlich, wie heterogen die „Bevölkerungsgruppe“ der Migranten ist. Es handelt sich um Menschen aus unterschiedlichen Herkunftsländern und Generationen mit verschiedensten Migrationsgeschichten, Bildungsniveaus, Aufenthaltsstatus und sozialen Lebenswelten.
Kultur. Der Begriff Kultur steht hier für einen Hintergrund aus etablierten und über Generationen hinweg übernommene Überlieferungen, Wertevorstellungen, Glaubenssätzen und Haltungen. Als ein Beispiel hierfür sollen die Konzepte von „namus“ und „seref“ genannt werden, die auch in Deutschland unter den Menschen mit türkischem Migrationshintergrund weitverbreitet sind [87]. Der Begriff namus beschreibt in diesem $\mathrm{Zu}-$ sammenhang die individuelle sexuelle Integrität einer Frau [4, 26, 30]. Seref dagegen umschreibt das Ansehen einer Familie innerhalb der Community, das vom Verhalten aller Familienmitglieder abhängt [82]. Traditionell sollen Töchter und Frauen die Tugendhaftigkeit und das Ansehen aller Männer innerhalb und außerhalb der Familie bewahren und die Sitten, Gebräuche und die Reinlichkeit unter der sozialen Kontrolle der Community leben [40, 41]. Damit werden in diesen Konzepten Frauen für den Erhalt von namus und seref verantwortlich gemacht.

Individuelle Modifikation. Die Konzepte über namus und seref zählen zu kulturspezifischen Faktoren, die das Denken, Fühlen und Handeln der Betroffenen beeinflussen [34, 72], sodass der Einzelne sie übernimmt und abhängig von den Kontextfaktoren wie Lebensraum, Alter, Geschlecht, Religion, Bildung und Akkulturation modifiziert [25, 72].

Die kulturellen Kontexte befinden sich in einem dynamischen Prozess.

Als Ausdruck eines dynamischen Prozesses kann es durchaus üblich sein, dass innerhalb einer Gruppe (z.B. in verschiedenen Migrantengenerationen) unterschiedliche Erklärungsmodelle und Behandlungserwartungen im Hinblick auf körperliche und seelische Erkrankungen nebeneinander zu finden sind [48, 49, 53, 54, 59]. Diese können je nach kultureller Entwicklung, persönlichen Erfahrungen und Informationen aus dem sozialen Umfeld oder den Medien einem ständigen Wandel unterliegen [34].

\section{Cultural Formulation}

Im DSM-5 wird mit dem Kapitel über Cultural Formulation unterstrichen, dass es für die diagnostische Einschätzung und das klinische Vorgehen essenziell ist, den kulturellen Kontext der Krankheitserfahrung eines Patienten zu verstehen [24]. Als Instrument wird der Einsatz des Cultural Formulation Interviews (CFI) empfohlen. Das CFI kann besonders hilfreich sein bei Diagnoseschwierigkeiten aufgrund bedeutsamer 
Unterschiede des kulturellen, religiösen oder sozioökonomischen Hintergrunds von Behandler und Patient. Eine weitere Einsatzmöglichkeit besteht bei Unsicherheiten über die Übereinstimmung kulturell ausgeprägter Symptome mit diagnostischen Kriterien, bei Schwierigkeiten in der Beurteilung des Schweregrads oder der Beeinträchtigung durch die Erkrankung. Auch bei Uneinigkeit über die Behandlung sowie bei begrenzter Motivation und Behandlungsadhärenz des Patienten kann der Einsatz des CFI hilfreich sein [24].

Im DSM-5 wird das CFI als Diagnoseinstrument bei unterschiedlichem kulturellen Hintergrund empfohlen.

\section{Cultural Formulation Interview}

Im CFI bezieht sich Kultur auf folgende Inhalte:

- „...Werte, Orientierungen, Wissen und Gebräuche, die Individuen aus ihrer Zugehörigkeit zu unterschiedlichen sozialen Gruppen ableiten (z. B. ethnische Gruppen, Glaubensgemeinschaften ...)

- Aspekte des Hintergrunds eines Patienten, Entwicklungserfahrungen und aktuelle soziale Kontexte, die seine Perspektive, wie geografische Herkunft, Migration, Sprache, Religion, sexuelle Orientierung oder Ethnizität beeinflussen können

- Einfluss der Familie, Freunde und anderer Community-Mitglieder (soziales Netzwerk des Patienten) auf die Krankheitserfahrung des Patienten“ [24, S. 1029]

\section{Zugangs- und Inanspruch- nahmebarrieren}

In Deutschland stehen nur wenige Studiendaten zur Inanspruchnahme psychiatrisch-psychotherapeutischer Angebote durch Migranten zur Verfügung [39, $51,52,57]$. Gezeigt wurde z. B., dass psychosomatisch erkrankte Migranten mit spanischem und italienischem Hintergrund häufiger Unterstützung in der Familie und im sozialen Umfeld suchen, während Personen mit russischem Hintergrund sich mehr an selbst umzusetzenden Maßnahmen (Hausmittel) orientieren [8].

Zugangsbarrieren. Personen mit russischem und türkischem Hintergrund geben sprachliche Probleme als wichtiges Hindernis für eine gezielte Inanspruchnahme an. Die Autoren betonen, dass bei türkeistämmigen Personen die Einschätzung, Fachkräfte wüssten zu wenig über die türkische Kultur, das wichtigste Hindernis ist. Auch die subjektive Einschätzung der Deutschkenntnisse und das subjektive Wohlbefinden in Deutschland haben einen Einfluss auf die Ausprägung der erlebten Hindernisse [7, 8].

Unterschiedliche Aspekte wie fehlende Informationen bezüglich der Versorgungsmöglichkeiten im deutschen Gesundheitssystem, aufenthaltsrechtliche Faktoren [29], Verständigungsschwierigkeiten und „kulturelle“ Missverständnisse [32, 34, 69, 71, 84], die den Weg in das Versorgungssystem verhindern bzw. zumindest verzögern, gelten als weitere Barrieren.

Kulturelle und kommunikative Barrieren können zu Problemen von Unter-, Über- und Fehlversorgung von Migranten führen [15].

Auch Diskriminierungserfahrungen [39], Stigmatisierung und Scham könnten weitere Ursachen für eine Unterversorgung, zumindest von einigen Gruppen von Migranten mit psychischen Störungen, sein [64, 83].

Stationäre Versorgung. Zur stationären Inanspruchnahme führten Schouler-Ocak et al. eine bundesweite repräsentative Umfrage in 131 psychiatrisch-psychotherapeutischen Kliniken durch [84]. Die Autoren stellten fest, dass $17 \%$ der Patienten der untersuchten Einrichtungen Migranten waren. Diese Erhebung erfolgte 2006, sodass hier die Daten aus dem Mikrozensus 2005 als Vergleichsdaten herangezogen werden konnten [93]. 2005 waren 18,6\% der Gesamtbevölkerung Migranten. Dies scheint dem Anteil der Migranten zu entsprechen, die sich in der stationären psychiatrischen Behandlung befanden. Bei genauer Auswertung der Daten zeigte sich jedoch, dass Migranten gerade in den Abteilungen für Psychotherapie/Psychosomatik (4,5\%), Gerontopsychiatrie (9,2\%) und Kinder- und Jugendpsychiatrie $(11,4 \%)$ sowie Sucht/Reha $(11,4 \%)$ unter-, in der Forensik (27,2\%) und der Abteilung für Abhängigkeitserkrankungen $(21,8 \%)$ überrepräsentiert waren [51].

Ambulante psychiatrische Versorgung. In einer bundesweit in psychiatrischen Institutsambulanzen (PIA) durchgeführten Untersuchung hatten 32,5\% der Patienten einen Migrationshintergrund und erhielten häufiger eine Diagnose aus dem Bereich der neurotischen, Belastungs- und somatoformen Störungen im Vergleich zu Patienten ohne Migrationshintergrund. Insbesondere Patienten mit türkischem Migrationshintergrund wurden deutlich häufiger der Diagnosegruppe der affektiven Störungen (F3) zugeordnet. Die 
hohe Inanspruchnahme durch Migranten weist darauf hin, dass offenbar die niederschwelligen und multiprofessionellen Angebote der PIA ihren Bedürfnissen besser gerecht werden [85].

\section{Interkulturelle Öffnung}

Mithilfe der interkulturellen Öffnung soll erreicht werden, dass ein gleichwertiger und gleichberechtigter Zugang zu Angeboten der Regelversorgung des Gesundheitssystems sowie eine kultursensible adäquate Diagnostik und Therapie für Migranten gewährleistet wird. Die interkulturelle Öffnung in Deutschland wurde erstmals von verschiedenen psychiatrischen Fachgesellschaften in den sog. „Sonnenberger Leitlinie$n$ “ zusammengefasst [61].

Die interkulturelle Öffnung wird von psychiatrischen Fachgesellschaften und auch von der Politik gefordert [22, 23].

Wie im Positionspapier der Deutschen Gesellschaft für Psychiatrie und Psychotherapie, Psychosomatik und Nervenheilkunde (DGPPN) hervorgehoben wird, ist eine nachhaltige und alltagstaugliche Verbesserung der medizinischen Versorgung von Migranten im ambulanten und stationären Bereich nur gewährleistet, wenn die Gesundheitsinstitutionen verbindliche Aktivitäten zur interkulturellen Öffnung entwickeln und diese personell zuordnen $[23,55]$. Penka et al. stellen fest, dass das Konzept der interkulturellen Öffnung in die Organisations-, Personal- und Qualitätsentwicklung von Institutionen einfließen sollte [70].

\section{Tipp für die Praxis}

Die interkulturelle Öffnung ist eine Leitungsaufgabe und somit eine Top-down-Maßnahme [23]. Dies ist nur möglich, wenn es gelingt, die institutionellen Strukturen zu adaptieren [33].

Nur wenige Studien befassen sich in Deutschland mit dem Stand der interkulturellen Öffnung. Einheitliche Messinstrumente zur Bestimmung der Umsetzung der interkulturellen Öffnung liegen nicht vor. Das wohl umfassendste Instrument ist von Penka et al. entwickelt worden [69].

Nationale Untersuchungen zum Stand der Umsetzung der interkulturellen Öffnung sind dringend nötig.

\section{Migrations-, Migranten-, Integrations- verantwortliche}

Im Integrationsplan wird die Implementierung eines Migrations- oder Integrationsbeauftragten als ein Schritt zur Umsetzung des Diversity Managements empfohlen [22]. Dieser kann aus allen Berufsgruppen stammen und dabei helfen, dass die besonderen Bedürfnisse von Patienten mit Migrationshintergrund berücksichtigt werden und die behandelnden und pflegenden Mitarbeiter die psychiatrisch-psychotherapeutische Versorgung dieser Patienten leichter bewältigen können $[23,55]$.

Je nach spezifischer Situation der jeweiligen Einrichtungen, der Patientenstruktur, dem Versorgungsschwerpunkt und den zur Verfügung stehenden Ressourcen können die konkreten Aufgabenbereiche des Integrationsbeauftragten wechseln $[23,55]$. Folgende Bereiche fallen in sein Gebiet:

- Koordination einzelner Maßnahmen

- Dolmetschersystem

- Aus-, Fort- und Weiterbildung

- Öffentlichkeitsarbeit

- Vernetzung und Durchführung von Beteiligungsprozessen

Für den Integrationsbeauftragten ergibt sich ein breites Aufgabenspektrum.

Der Integrationsbeauftragte sollte in das Qualitätsmanagement eingebettet werden, damit die vertikale und horizontale Kommunikation und das Monitoring migrationsspezifischer Daten gewährleistet werden können [23]. Zudem zählen zu seinen Aufgaben die Teilnahme an Gremien und Arbeitskreisen (z. B. QMTeam), in denen Pläne und Konzepte zur Zukunft der Einrichtungen entwickelt werden $[23,55]$. So können organisatorisch gesicherte und kontinuierliche Absprachen mit der Betriebsleitung regelmäßig in den Prozess der interkulturellen Öffnung fließen [55].

\section{Interkulturelle Kompetenz}

Pfeiffer beschreibt Kultur als einen „Komplex, der überlieferte Erfahrungen, Vorstellungen und Werte umfasst sowie gesellschaftliche Ordnung und Verhaltensregeln“ [72, S.10]. Tseng dagegen sieht in der Kultur einen ständigen Veränderungsprozess, in dem die Kultur das Verhalten der Menschen prägt und gleichzeitig von den Ideen und dem Verhalten der Mitglieder einer Kultur geformt wird [97]. Kumbruck und Derboven 
umschreiben Kultur als etwas, das im sozialen Diskurs um Bedeutungen entsteht, wobei Sinn und Wert von kulturellen Traditionen, Praktiken und Erfahrungen ausgehandelt werden [56]. Daraus resultiert die Annahme, dass die interkulturelle Kompetenz eine Komponente der sozialen Kompetenz ist.

Offenheit und respektvolle Neugier. Grosch und Leenen stellen fest, dass Wahrnehmen, Urteilen und Handeln immer auch kulturell bedingt sind [28]. Für Oestereich und Hegemann sind Offenheit, Interesse und respektvolle Neugier auf Ungewohntes die Grundpfeiler interkultureller Kompetenz [67]. Kirmayer et al. sehen in der interkulturellen Kompetenz die Fähigkeit, mit Sprach- und Kulturvermittlern arbeiten zu können, Idioms of Distress (kulturspezifische Ausdrucksmuster) zu erkennen und Krankheitsverständnisse und Behandlungserwartungen der Patienten sowie das Ausarbeiten kulturell passender Erklärungen und Behandlungsangebote zu beachten [46].

Die interkulturelle Kompetenz setzt kulturelles Wissen und Kompetenzen wie Umgang mit Sprachund Kulturmittlern und eine entsprechende Haltung voraus $[74,88]$.

\section{Tipp für die Praxis}

Interkulturelle Kompetenz ist dort erforderlich, wenn Behandler und Patient unterschiedliche kulturelle Hintergründe haben, um den Bedürfnissen des Patienten gerecht werden und eigene Vorurteile oder Befangenheiten ablegen zu können [12-14, 88]. Carpenter-Song et al. empfehlen, offen zu sein für neue ungewöhnliche Beschwerden und Schilderungen [29].

Bedeutung für die Bildungssysteme. Mit zunehmender Globalisierung nimmt die Bedeutung der interkulturellen Kompetenz zu und sollte unbedingt in die Aus-, Fort- und Weiterbildung aller im Gesundheitssystem Tätigen integriert werden [66]. Es handelt sich dabei um einen Prozess, der nach Implementierung fortlaufend unter Monitoring weiterentwickelt werden sollte $[47,88]$.

\section{Arbeit mit Dolmetschern (Sprach- und Kulturmittlern)}

Koch et al. berichten, dass in 12 großen psychiatrischpsychotherapeutischen stationären Einrichtungen die multiprofessionellen Behandlerteams angaben, im
Kontakt mit Migranten Verständigungsprobleme zu haben. Diese wurden mit $27 \%$ als sprachgebunden, mit $38 \%$ als kulturgebunden und mit $44 \%$ als kultur- und sprachgebunden quantifiziert [51].

Offenbar sind Verständigungsprobleme im Behandlungssetting mit Migranten sprachund kulturgebunden.

Ohne eine Verständigung ist eine Behandlung nicht möglich. Behandler müssen auch für Personen mit geringen Kenntnissen der deutschen Sprache eine angemessene Behandlung gewährleisten. Entsprechend einer Expertise, erstellt im Auftrag des BAMF, liegt der Anteil von Migranten mit schlechten Deutschkenntnissen bei rund 20\% [63]. In diesem Zusammenhang sind Kenntnisse im Umgang mit Dolmetschern (Sprach- und Kulturmittlern) und deren Verfügbarkeit von großer Bedeutung. Dabei kann es sich z.B. um Gemeindedolmetscherdienste wie in Berlin handeln, es sind aber in größeren Kliniken auch Dolmetscherdienste von bilingualem Fachpersonal denkbar [5, 55, 105].

\section{Tipp für die Praxis}

Problematisch ist das Hinzuziehen von ungeschultem Personal oder von Angehörigen als Laien-Übersetzer [23]. Der Einsatz von qualifizierten Sprach- und Kulturmittlern sollte routinemäßig dann erfolgen, wenn Verständigungsprobleme bestehen.

Die Verständigung bildet das Hauptarbeits-

instrument der Psychiatrie und Psychotherapie.

Eine muttersprachliche Behandlung scheitert in der Regel an einem Mangel an qualifizierten Muttersprachlern und an Sprach- und Kulturmittlern. Erschwert wird die Situation auch dadurch, dass ausländische Ärzte deutliche Schwierigkeiten haben, eine außerhalb Deutschlands erworbene Berufserlaubnis anerkennen zu lassen [23]. Das DGPPN-Positionspapier fordert, dass Leitfäden für den professionellen Einsatz von Sprach- und Kulturmittlern nicht nur von professionellen Dolmetschern, sondern auch von den Professionellen im Gesundheitssystem verinnerlicht werden $[16,23]$. Dadurch können Missverständnisse vermieden und die Möglichkeiten einer dolmetschergestützten Diagnostik und Behandlung genutzt werden $[16,65]$. 


\section{Epidemiologische Aspekte und Besonderheiten psychiatrischer Störungsbilder bei Migranten}

Im Vergleich zu anderen europäischen Ländern liegt in Deutschland bisher nur eine kleine Zahl an bevölkerungsbezogenen epidemiologischen Untersuchungen zum Auftreten und Verlauf von psychiatrischen Störungen in verschiedenen Migrantengruppen bzw. kulturellen Minderheiten vor [6]. Die wenigen bevölkerungsbezogenen Daten stammen sonst aus Untersuchungen, deren eigentliches Ziel nicht die Erfassung von Prävalenzen und Inzidenzen psychiatrischer Diagnosen in Migrantengruppen war, sondern die Variablen zur nationalen oder kulturellen Herkunft der Probanden erfassten und somit eine zusätzliche Auswertung nach Migrationshintergrund erlauben [2, 3, 6, 27]. Die gezielte Untersuchung von Kindern und Jugendlichen mit Migrationshintergrund war und ist jedoch Ziel der Kinder- und Jugendgesundheitssurveys (KiGGS) [78]. Eine Darstellung zu den Störungsgruppen, zu denen bislang die meisten Informationen vorliegen, erfolgt unten.

Bislang liegen Prävalenz und Inzidenzdaten v. a. für affektive Störungen und psychotische Störungen vor.

\section{Abhängigkeitserkrankungen (ICD-10: F1)}

Bevölkerungsrepräsentative Daten zu Abhängigkeitserkrankungen bzw. schädlichem Konsum von Alkohol und psychotropen Substanzen in Migrantengruppen fehlen für Deutschland bislang weitgehend [76]. Es wird angenommen, dass insbesondere Menschen mit türkischem und russischem Migrationshintergrund besonders von Abhängigkeitserkrankungen betroffen sind [102].

In den vergangenen Jahren beschäftigten sich jedoch zunehmend mehr Studien in Deutschland mit dieser Thematik in Migrantengruppen. So zeigte z.B. eine Analyse von Daten der Deutschen Suchthilfestatistik des Instituts für Therapieforschung München (IFT) der Jahre 2002 -2012, dass Männer mit Migrationshintergrund entsprechend ihrem Bevölkerungsanteil in der ambulanten Suchthilfe vertreten waren, Frauen mit Migrationshintergrund, mit Ausnahme der Kokainabhängigkeit, jedoch unterrepräsentiert waren. Des Weiteren waren Männer mit Migrationshintergrund deutlich überrepräsentiert, wenn sie eine der folgenden
Diagnosen aufwiesen: Cannabismissbrauch, Opioidabhängigkeit, Kokainabhängigkeit und pathologisches Spielen. Insgesamt waren Personen mit eigener Migrationserfahrung allerdings unterrepräsentiert [79]. Eine von Weilandt et al. bislang unveröffentlichte Untersuchung zeigte, dass im Jahr 2000 in Nordrhein-Westfalen männliche nicht deutsche Staatsangehörige häufiger wegen den Konsumfolgen von psychotropen Substanzen stationäre Rehabilitationsangebote wahrnahmen als wegen Alkoholabhängigkeit [104]. Frauen mit nicht deutscher Staatsangehörigkeit nahmen dagegen allgemein seltener als deutsche Frauen Rehabilitation in Anspruch [104].

\section{Schizophrenie und wahnhafte Störungen (ICD-10: F2)}

Eine von Schouler-Ocak et al. durchgeführte deutschlandweite Erhebung der stationär-psychiatrischen Versorgung zeigte, dass insbesondere schizophrene Psychosen und wahnhafte Störungen häufiger bei Migranten diagnostiziert wurden [84]. Gleichzeitig berichteten die Autoren, dass viele der befragten Institutionen häufige Kommunikationsschwierigkeiten, wie z.B. Sprachbarrieren, angaben, durch die die Einschätzung der Symptomatik teilweise erschwert oder beeinträchtigt sein könnte. Daten aus bevölkerungsbezogenen epidemiologischen Studien zu Migranten in diesem Bereich fehlen in Deutschland fast gänzlich, jedoch sprechen die Ergebnisse von einigen internationalen Untersuchungen aus den Niederlanden, Großbritannien oder Dänemark dafür, dass Migranten, insbesondere der 2. Generation bzw. Personen, die sehr früh migriert sind, unter einem größeren Risiko für das Auftreten einer psychotischen Störung stehen [42, 64, 90 , $98-100]$.

\section{Affektive und somatoforme Störungen, Angsterkrankungen (ICD-10: F3 \& F4)}

Bevölkerungsrepräsentative Studien. Bislang liegen zu dieser Diagnosegruppe die meisten Daten sowohl aus der Allgemeinbevölkerung (s.o.) als auch aus der ambulanten und stationär-psychiatrischen Versorgung vor. So fanden Bermejo et al. in einer Folgeauswertung des Bundesgesundheitssurveys aus den Jahren 1998/ 99 bei Migranten eine höhere 4-Wochen-, 12-Monatssowie Lebenszeitprävalenz bei affektiven und somatoformen Störungen [6]. Einschränkend muss jedoch erwähnt werden, dass die Untersuchung nicht speziell den Einschluss von Migranten zum Ziel hatte und keine 
Unterscheidung nach Migrationshintergrund erfolgte, sondern nur unterschieden wurde zwischen nicht in Deutschland geborenen Personen ohne deutsche Staatsbürgerschaft und Personen, die in Deutschland geboren wurden und die deutsche Staatsbürgerschaft besaßen [6].

Eine weitere bevölkerungsrepräsentative Studie von Glaesmer et al. untersuchte die Frage der Häufigkeit einzelner psychiatrischer Störungen bei Migranten in Deutschland und fand im Gegensatz zu Bermejo et al. keinen Unterschied zwischen Migranten und Nichtmigranten in der 2- bzw. 4-Wochen-Prävalenz depressiver und somatoformer Symptome (2-Wochen-Prävalenz), bei generalisierter Angstsymptomatik und posttraumatischen Belastungsstörungen (PTBS) [6, 27]. Auch diese Studie richtete sich nicht spezifisch an Migranten. Zudem bestand keine deutsche Vergleichsgruppe. Eine Unterscheidung nach Migrationshintergrund erfolgte ebenfalls nicht. Die Bestimmung der Prävalenzen erfolgte im Gegensatz zu der erstgenannten Studie mittels Screeninginstrumenten [27].

Depression. Aichberger et al. fanden in einer Auswertung der deutschen Daten einer bevölkerungsrepräsentativen Untersuchung zu Gesundheit, Altern und sozioökonomischer Lage älterer Menschen in Europa (Survey of Health, Ageing, and Retirement - SHARE) bei Migranten (in diesem Fall nur Personen mit eigener Migrationserfahrung) eine höhere Prävalenz depressiver Symptome (28 vs. 19\%) als bei Nichtmigranten [2]. Auch in dieser Studie erfolgte keine gezielte Rekrutierung von Migranten und die Bestimmung depressiver Symptome mittels eines Screeninginstruments.

Viele Studien befassen sich nicht explizit mit der Migrantenpopulation.

Versorgungsstudien. In Zusammenschau lassen die Ergebnisse der aufgeführten Studien keine eindeutigen Rückschlüsse zu, jedoch weisen Studien aus dem klinischen Versorgungsalltag eher darauf hin, dass Migranten in Deutschland häufiger eine affektive oder somatoforme Diagnose erhalten [6]. So fanden SchoulerOcak et al. in einer bundesweiten Erhebung zur Versorgungssituation von Migranten in Psychiatrischen Institutsambulanzen, dass insbesondere türkeistämmige Migranten deutlich häufiger die Diagnose einer affektiven Störung erhielten [85].

Metaanalyse. Eine Metaanalyse zum Zusammenhang zwischen Migrationshintergrund und Auftreten affektiver Störungen kam zum Schluss, dass aufgrund der aktuellen Datenlage bis auf den Verdacht, dass Migranten ein höheres Risiko für bipolare Störungen aufweisen könnten, eine abschließende Aussage zu weiteren affektiven Störungsbildern zurzeit nicht möglich ist [95].

\section{Suizid und Suizidversuche}

Eine Reihe von Untersuchungen in verschiedenen europäischen Ländern hat in den letzten 20 Jahren auf erhöhte Suizidversuchs- und Suizidraten in manchen Migrantengruppen hingewiesen [37]. Insbesondere Mädchen und junge Frauen (15-24 Jahre) zeigen ein erhöhtes Risiko [17, 18]. Daten aus Deutschland weisen einerseits auf eine erhöhte Suizidrate bei 10- bis 17jährigen türkeistämmigen Mädchen hin und andererseits auf erhöhte Suizidversuchsraten bei russischen, türkischen und polnischen Migranten und Migrantinnen $[60,75]$.

\section{Beschwerdepräsentation}

Neben der Frage nach Prävalenz, Inzidenz und dem Verlauf der einzelnen Störungen ist für den klinischen Alltag die Frage nach Besonderheiten der Symptompräsentation bei Migranten von wesentlicher Bedeutung. Mehrheitlich fanden Untersuchungen, dass insbesondere die initiale Darbietung von Beschwerden durch kulturelle Aspekte überlagert bzw. beeinflusst wird [44, 45], aber dass bei gezielter Befragung keine relevanten Unterschiede bestehen [92]. Dass die Kultur eine wichtige Rolle für die Beschwerdepräsentation im diagnostischen und therapeutischen Prozess spielt, wird in zahlreichen Publikationen berichtet [12-14].

\section{Belastungs- und Schutzfaktoren}

Eine Vielzahl von migrationsspezifischen Faktoren (z.B. ob eine Person selbst migriert ist, unter welchen Bedingungen und mit welchen Motiven) aber auch allgemeinen sozialen Faktoren (z. B. unter welchen sozialen Rahmenbedingungen jemand aufgewachsen ist) können gemeinsam mit individuellen biologischen Risiken zu einer Prädisposition für die Entwicklung psychiatrischer Störungen bei Migranten führen. Während unbestritten ist, dass die individuellen biologischen, insbesondere genetischen Risiken, sich zwischen Migranten und Nichtmigranten nicht wesentlich unterscheiden, könnten soziale Risiko- und Belastungsfaktoren in verschiedenen Migrantengruppen unterschiedlich verteilt sein [12, 36]. 


\section{Migrationsspezifische Faktoren}

\section{- Traumatische Erfahrungen vor oder während der Migration}

$\mathrm{Zu}$ den bedeutsamsten Belastungen bei Migranten mit eigener Migrationserfahrung zählen sicherlich traumatische Erfahrungen im Rahmen der Migration. Migranten mit Erfahrung von Flucht vor bewaffneter Auseinandersetzung im Rahmen von (Bürger-)Kriegen haben ein besonderes Risiko für das Auftreten von vorübergehenden, aber auch chronischen psychiatrischen Beschwerden [1]. So fanden Lindert et al. in einer Metanalyse von Studien zu depressiven Störungen und Angsterkrankungen bei Arbeitsmigranten und Flüchtlingen, dass Flüchtlinge eine doppelt so hohe Prävalenz bei depressiven Störungen sowie Angsterkrankungen aufwiesen ( $20 \%$ vs. $44 \%$ und $21 \%$ vs. $40 \%$ ) [58]. Zudem weisen Studien darauf hin, dass die Rate der Posttraumatischen Belastungsstörungen (PTBS) bei Flüchtlingen und Asylbewerbern im Vergleich zur Allgemeinbevölkerung um das 10-Fache erhöht ist [21].

\section{akkulturation und akkulturativer Stress}

Das Ankommen in einer neuen Gesellschaft, in einem neuen Land, erfordert eine hohe Anpassungsleistung von den ankommenden Menschen. Die sozialen und v.a. psychischen Belastungen, die spezifisch mit dieser Phase der Migration in Verbindung gebracht stehen, werden unter dem Begriff akkulturativer Stress zusammengefasst [10].

Akkulturation, der gegenseitige Austausch von kulturellen Normen und Traditionen von miteinander in stetigen Kontakt tretenden Personengruppen unterschiedlicher Kulturen, umfasst nach Berry 2 Dimensionen:

- die Aufrechterhaltung kultureller Werte und

- den Kontakt mit und die Teilhabe an der "neuen“ Gesellschaft [11].

In Abhängigkeit von der Nähe bzw. Ähnlichkeit von Verhaltensweisen und Werten können 4 sog. Akkulturationsstrategien abgeleitet werden [103]. Hier handelt es sich nicht um statische Strategien, sie sind immer wieder einer dynamischen Veränderung je nach Einflussfaktoren unterworfen [34]. Von den Akkulturationsstrategien wird zumeist die Marginalisierung mit höheren Raten von psychischer Belastung bzw. geringerer psychischer Gesundheit in Zusammenhang gebracht $[11,81]$.

\section{Die 4 Akkulturationsstrategien}

Integriert: Individuum teilt sowohl das kulturelle Normsystem der Ursprungs- als auch der neuen Kultur

Separiert: Individuum hat keine oder kaum Aspekte des Normsystems der neuen Kultur übernommen

Marginalisiert: Individuum ist sowohl von der Ursprungs- als auch von der neuen Kultur ausgegrenzt

Assimiliert: Individuum hat kulturelles Normsystem der neuen Kultur angenommen und teilt kaum oder keine Aspekte des Normsystems der Ursprungskultur mehr

nach Berry $[10,11]$

\section{Ethnische Diskriminierung und Rassismus}

Zahlreiche Befunde sprechen dafür, dass sich rassistisch motivierte Diskriminierung negativ auf körperliche und psychische Gesundheit auswirkt [35, 68, 98, 106]. Eine Vielzahl an Befunden bestätigt die gesundheitsbeeinträchtigenden Effekte in unterschiedlichen ethnischen Minderheiten bzw. Migrantengruppen [68]. In einer von Igel et al. in Deutschland durchgeführten Untersuchung berichteten 43,4\% der Migranten unabhängig vom Migrationshintergrund von häufigen Diskriminierungserfahrungen [39]. Die Autoren konnten außerdem in der Gruppe der GUS-stämmigen Migranten einen Zusammenhang mit seelischer Gesundheit zeigen.

\section{Aufenthaltsstatus und rechtliche Rahmen- bedingungen bei der Einwanderung}

Einige Untersuchungen konnten zeigen, dass die rechtlichen Rahmenbedingungen bei der Einbürgerung, die Dauer bis zur Bewilligung eines Aufenthaltsstatus sowie die Art des Aufenthaltstitels die psychische Belastung von Migranten verstärken können [73].

\section{- Verlust sozialer Netzwerke}

Gerade für Migranten mit eigener Migrationserfahrung, die ihre Heimat und oft Teile ihrer Familie zurücklassen mussten, kann der Verlust der gewohnten sozialen Netzwerke und damit von sozialer Unterstützung ein starker Belastungsfaktor sein $[91,96]$. Insbesondere Flüchtlinge, die meist keinen Abschied von Familie und Freunden nehmen konnten, können hierdurch v.a. zu Beginn deutlich mehr belastet sein. Auch in der Aufnahmegesellschaft sind offenbar soziale Faktoren der Migration und ihre Folgen wie Partizipation versus Ausschließung entscheidend für die Prävalenz z.B. psychotischer Erkrankungen [36]. 


\section{Präventive Angebote für Migranten}

Bisher ist die Zahl der spezifisch für bestimmte Migrantengruppen zugeschnittenen Präventionsangebote im Feld der Psychiatrie relativ gering.

MIMI-Projekt. An prominentester Stelle ist das MIMIProjekt (Mit Migranten für Migranten) zu nennen, das vom Ethno-Medizinischen Zentrum e.V. in Hannover entwickelt wurde. Das Projekt verbessert mittlerweile in verschiedenen deutschen Städten gesundheitsbezogenes Verhalten bei Migranten durch Schulungen von Schlüsselpersonen aus derselben Migranten-Community [107].

ISH-Projekt. Ein anderes Beispiel für ein Präventionsprogramm ist das ISH-Projekt (Interkulturelle Suchtprävention und Beratung), das muttersprachliche und kulturspezifische Aufklärungsangebote zu Drogen bereitstellt und ebenfalls Schlüsselpersonen (muttersprachliche Suchtpräventionsberater) einsetzt [107].

SPIMig-Projekt. Mit dem Thema suizidalen Verhaltens und der Verbesserung der Inanspruchnahme von Krisenhilfe im Rahmen von (suizidalen) Krisen beschäftigte sich das SPIMig-Projekt (Suizidprävention bei Berliner Frauen mit türkischem Migrationshintergrund) in Berlin, das neben einer bevölkerungsbezogenen Aufklärungskampagne eine muttersprachliche Krisenhotline und Schlüsselpersonen aus der Community mit gutem Kontakt zur Zielgruppe, die speziell dafür geschult wurden, einsetzte [86].

PRÄALMI-Projekt. Bei dem Projekt „Primärprävention alkoholbezogener Störungen bei älteren Migrantinnen und Migranten - Entwicklung und Evaluation eines transkulturellen Präventionskonzeptes“ (PRÄALMI) werden kulturelle Normen und Werte, migrationsbezogene Faktoren, spezifische Zugangsbarrieren im Suchthilfebereich sowie die Förderung interkultureller Kompetenz in Regeldiensten der Suchthilfe unterstützt [9].

\section{Kulturelle Aspekte der Psychopharmakologie}

Die Ethnopsychopharmakologie ist in Deutschland bislang ein noch zu wenig beachtetes Gebiet. Mit der Zunahme von Migranten aus verschiedenen Teilen der Welt rückt das Thema der unterschiedlichen Pharma- kogenetik mehr und mehr in den Fokus. Als genetisch unterschiedliche Hauptpopulationen werden nach Ruiz genannt:

- Kaukasier

- Mongoloide

- Negroide

- australische Aborigines

- San (Buschmänner) [80]

Die Pharmakogenetik, die Lehre von den erblichen Ursachen individueller Unterschiede in den Arzneimittelwirkungen und Nebenwirkungen, beschreibt z.B. genetische Unterschiede von Arzneimittelrezeptoren, Enzymen, die am Metabolismus beteiligt sind (z. B. CYP 450), und Proteine, die am Transport von Pharmaka beteiligt sind (z. B. P-Glykoprotein) [77].

\section{Genetisch verschiedene \\ Metabolisierungsgruppen}

Nach Schwab et al. werden 4 Metabolisierungstypen unterschieden [89]:

- extrem langsame Metabolisierer

- langsame Metabolisierer

- normale Metabolisierer

- ultraschnelle Metabolisierer

Über das Cytochrom-P450-Isoenzym 2C19 werden die Wirksubstanzen Moclobemid, Diazepam, Clomipramin, Amitriptylin und Imipramin abgebaut. Hier sind 3-5\% der Kaukasier und 15-20\% der Asiaten langsame Metabolisierer [89]. Dieses Beispiel macht deutlich, dass bei manchen Migrantengruppen auch pharmakogenetische Faktoren zu beachten sind. Aus Unwissenheit können falsche Substanzen eingesetzt oder Dosierungen nicht angepasst werden.

\section{Fazit und Perspektiven}

Mehr als 16,5 Mio. Migranten (21\% der Gesamtbevölkerung) haben inzwischen ihren Lebensmittelpunkt in Deutschland. Es ist davon auszugehen, dass in den kommenden Jahrzehnten die Zahl der Migranten noch steigen wird. Das medizinische Versorgungssystem muss sich auf diese sehr heterogene Bevölkerungsgruppe mit ihren unterschiedlichen Biografien, Schutzund Belastungsfaktoren vorbereiten. Gesundheits- und Krankheitskonzepte sowie Behandlungserwartungen sind je nach kulturellem Hintergrund, traditionellen Werten, persönlichen Erfahrungen und sozialen Lebenswelten einem ständigen Wandel unterworfen [12-14, 24, 34]. Zugangsbarrieren können besser 
überwunden bzw. gemindert werden, wenn die jeweiligen sozialen Lebensumstände von Migranten beachtet, die individuellen Erklärungsansätze bezüglich Erkrankungen und ihre Behandlungserwartungen berücksichtigt werden [12-14, 24, 34, 69, 71].

Das medizinische Versorgungssystem ist noch nicht gut gerüstet für die speziellen Bedürfnisse von Migranten.

Soziale Exklusion, niedrige Bildung, schlechte ökonomische Lage und niedrige ethnische Dichte sind Belastungsfaktoren, die bei Migranten zu einem höheren Erkrankungsrisiko für bestimmte Störungen führen [36, 39, 64, 91, 95, 100, 101]. Neben diesem erhöhten Erkrankungsrisiko können Migranten durch zahlreiche Zugangs- und Inanpruchnahmebarrieren wie aufenthaltsrechtliche Faktoren [29], Verständigungsschwierigkeiten und „kulturelle“ Missverständnisse [32, 34, 69, 71, 84], Diskriminierungserfahrungen [39], Stigmatisierung und Scham $[64,83]$ benachteiligt sein. Um die Barrieren zu senken, sind interkulturelle Öffnung der Einrichtungen und interkulturelle Kompetenz aller Mitarbeiter erforderlich. Doch nach wie vor ist die interkulturelle Öffnung der Institutionen im Gesundheitssystem mit Einrichtung eines Migrations- bzw. Integrationsbeauftragten nicht vorangekommen $[55,61,69,70]$.

Auch der Erwerb der interkulturellen Kompetenz der Mitarbeiter aller Berufsgruppen entwickelt sich zögerlich [12-14, 28, 46, 47, 66, 74]. Oftmals ist der Einsatz eines Übersetzers erforderlich, da sonst keine ausreichende Kommunikation möglich ist $[12,13,55,105]$.

Die interkulturelle Öffnung der Institutionen des Gesundheitssystems ist noch wenig entwickelt.

\section{Kernaussagen}

Die sehr heterogene Gruppe der Migranten nimmt stetig zu und taucht mehr und mehr in der Versorgung auf. Mithilfe des Cultural Formulation Interviews (CFI) können ihre Gesundheits- und Krankheitskonzepte sowie Behandlungserwartungen und Belastungsfaktoren erfasst werden. Interkulturelle Öffnung der Einrichtungen und interkulturelle Kompetenz der Mitarbeiter sowie der reguläre Einsatz von profes-
Auch ethnopharmakotherapeutische Einflussfaktoren müssen bei der Versorgung von Migranten berücksichtigt werden [38, 77, 80, 89]. Menschen mit Migrationshintergrund haben besondere gesundheitliche, psychosoziale und ökonomische Belastungen [76]. Kulturelle Aspekte, migrationsspezifische und soziale Faktoren beeinflussen die Prävalenz, Manifestation und den Verlauf psychischer Erkrankungen bei Migranten [12].

Zusammenfassend ist zu hoffen, dass die in diesem Beitrag aufgeführten Aspekte der Versorgung von Migranten regelhaft in die Aus-, Fort- und Weiterbildungen integriert werden. Denn in Anbetracht der großen Bevölkerungsgruppe können diese Versorgungslücken nicht mehr länger hingenommen werden.

\section{Über die Autorinnen}

\section{Meryam Schouler-Ocak}

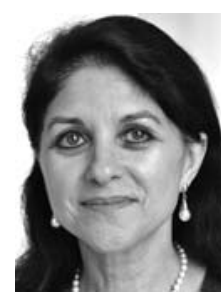

PD Dr. med. Ltd. Oberärztin der Psychiatrischen Universitätsklinik der Charité im St. Hedwig Krankenhaus Berlin. Leitung Forschungsbereich Interkulturelle Migrations- und Versorgungsforschung, Sozialpsychiatrie an der Charité. Leiterin des Referats Interkulturelle Psychiatrie und Psychotherapie, Migration der Deutschen Gesellschaft für Psychiatrie und Psychotherapie, Psychosomatik und Nervenheilkunde (DGPPN). Chair of Section of Cultural Psychiatry of the European Psychiatrists Association (EPA).

\section{Marion Christina Aichberger MSc}

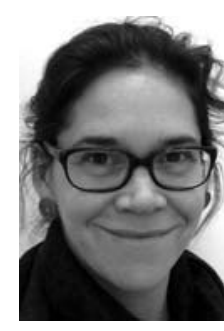

Dr. med. Ärztin an der Klinik für Psychiatrie und Psychotherapie, Campus Mitte, Charité - Universitätsmedizin Berlin. Wissenschaftliche Mitarbeiterin im Forschungsbereich Interkulturelle Migrations- und Versorgungsforschung, Sozialpsychiatrie an der Charité.

Interessenkonflikt: Die Autorinnen geben an, dass in Bezug auf diesen Beitrag kein Interessenkonflikt besteht.

\section{Korrespondenzadresse}

PD Dr. med. Meryam Schouler-Ocak

Psychiatrische Universitätsklinik der Charité

St. Hedwig-Krankenhaus

Große Hamburger Straße 5-11

10115 Berlin

E-Mail: meryam.schouler-ocak@charite.de 


\section{CME-Fragen}

\section{CME。thieme.de}

\section{CME-Teilnahme}

- Viel Erfolg bei Ihrer CME-Teilnahme unter http://cme.thieme.de

- Diese Fortbildungseinheit ist 12 Monate online für eine CME-Teilnahme verfügbar.

- Sollten Sie Fragen zur Online-Teilnahme haben, unter http://cme.thieme.de/hilfe finden Sie eine ausführliche Anleitung.

\section{1}

Welche Aussage zum Gesundheitszustand der Migranten in Deutschland trifft zu?
A Der Gesundheitszustand ist meist besser als der von Einheimischen.

B Es findet sich eine höhere Nutzung von Angeboten zur Prävention.

C Die Vorsorgeuntersuchungen werden gut angenommen.

D Die Notfallambulanzen werden insgesamt weniger beansprucht.

E Bei Flüchtlingen und Asylbewerbern ist die Rate der Posttraumatischen Belastungsstörung (PTBS) im Vergleich zur Allgemeinbevölkerung deutlich erhöht.

\section{2}

Welche kulturellen Faktoren können die Compliance positiv beeinflussen?
A unterschiedliche Behandlungserwartungen
B übereinstimmende Krankheitskonzepte
C religiöse Verbote (z. B. Schweinefleisch)
D mangelnde verbale Verständigung
E autoritäre Arzt-Patient-Beziehung

\section{3}

Ab welchem Einwanderungsjahr wird nach der offiziellen Definition in Deutschland von einem Menschen mit Migrationshintergrund gesprochen?
A 1918
B 1949
C 1955
D 1989
E 2001

Wie lautet die genaue Definition für Menschen mit Migrationshintergrund in Deutschland?
A Ausländer, die von außerhalb der EU zugewandert sind
B Eingebürgerte mit ausländischer Herkunft
C Selbsteingewanderte, deren Kinder und Kinder derjenigen, die als Ausländer in Deutschland geboren wurden
D Arbeitnehmer, die mit einer Green Card nach Deutschland kamen und deren Angehörige
E Migranten, die nicht die deutsche Staatsbürgerschaft haben

Ein Patient mit arabischem Migrationshintergrund ohne Deutschkenntnisse kommt zur Behandlung zur Klinik. Wie gehen Sie vor?

\section{6}
A Begleitperson übersetzen lassen
B Reinigungskraft oder andere Mitarbeiter zur Übersetzung bitten
C Übersetzung nicht erforderlich, Kommunikation wird irgendwie gehen
D Sprach- und Kulturvermittler einsetzen
E Versuche, mit dem Patienten schriftlich zu kommunizieren
A muttersprachlicher Arzt
B therapeutisches Spezialangebot
C Einsatz von professionellen Kultur- und Sprachmittlern
D Informationslücken beim Patienten
E Aufklärung in der Muttersprache 


\section{CME-Fragen vesorgang yon Muganaten}

\section{7}

Was ist keine Akkulturationsstrategie nach Berry?
A Integration
B Assimilation
C Segregation
D Marginalisierung
E Remigration

\section{8}

Wo treten keine genetischen Unterschiede auf?
A Arzneimittelrezeptoren
B Enzymen
C Seren
D Proteinen
E Arzneimittelwirkungen

\section{9}

Was zählt nicht zu den Metabolisierungstypen?
A extrem langsame Metabolisierer
B langsame Metabolisierer
C normale Metabolisierer
D ultraschnelle Metabolisierer
E Blitzmetabolisierer

Was können Zugangsbarrieren für Migranten sein?
A Patienten mit Migrationshintergrund sind gut über die Strukturen des Gesundheitssystems informiert.

B Mitarbeiter der Kliniken verfügen über ausreichende Kenntnisse zu Vorstellungen von Erkrankungen der Patienten mit Migrationshintergrund.

C Mitarbeiter und Patienten mit Migrationshintergrund können nicht miteinander kommunizieren.

D Interkulturell kompetente Mitarbeiter stehen zur Verfügung..

E Die interkulturelle Öffnung einer Klinik hängt davon ab, ob Mitarbeiter mit Migrationshintergrund dort arbeiten wollen.

Literatur

Die Literatur zu diesem Beitrag finden Sie unter

http://dx.doi.org/10.1055/s-0041-100492 